blocks which can be tuned to the specific needs of each installation. This is because important progress in hardware usually makes a low-level language unnecessary (even "real" programmers no longer write code in hexadecimal). More people also realize that designing a new menu facility (a graphic display to help an operator interact with the experiment) is a waste of time. Attention should be directed instead to ensuring requirements are agreed upon and understood. Computer-assisted software engineering (CASE) tools, although not yet fully mature, are being used more and more.

However, it is also clear that the implementation of standards is governed by powerful market forces. Aside from wishful thinking, we users have not come together to specify requirements, although the achievements of the EPS control systems group (EPCS) constitute the essential first step in a sharing of expertise.

\section{Porting}

CODAS is presently being modified to assure its availability, reliability and performance for the years to come by porting to a UNIX operating environment. The move to UNIX (V.4) for the operating system, Ethernet 802.3 for communication, VME, Fortran 77 and $C$ as programming language and workstations for the man-machine interfaces has highlighted the importance of software portability because the effort to implement the move estimated to be as large as 35 man-years.

It is beyond the scope of this paper to justify the above choices: some were dictated by the need to minimize the porting effort and budget; others followed from making CODAS as independent as possible of the fortunes of a single manufacturer. For the sake of compatibility with our old 200 Gbyte of data, and to leave the door open to a mixed system (old and new), we were also obliged to choose the the larger vendors [8]. We are also unable to use CASE tools as we are moving more than two million lines of existing code rather than designing a new system.

The advent of de facto standards since 1979 and the availability of certain tools has made some parts of our software not only obsolete but unnecessary. We are therefore using, whenever feasible, public domain software although we shall have to continue to choose between products coming from competing groups (e.g. the Free Software Foundation and UNIX [9]) and between differing approaches to high level tools for workstations.

\section{Conclusions}

I am not tacitly advocating that a universal standard should apply to all computers and all computer-assisted control systems as I have been in research too long not to realize that progress comes from multiple approaches. Market forces, more- over, will continue to force changes and existing standards will be superseded. Nonetheless, we shall still have the opportunity to minimize the effort expended on design and implementation because useful and well-supported standards are now available.

Strong managerial support of the EPCS's efforts to rationalize some of the development of control systems for physics experiments could reduce the costs of "closed-shop" projects and provide better tools and procedures to all laboratories. But to reap its full benefits, this support will have to be substantial and of a continuing nature.

\section{REFERENCES}

[1] Gibson A., Proc. EPS-8 General Conf., Amsterdam, 1990 (to be published).

[2] Fusion Technology 11.1 (1987) 1.

[3] JET Joint Undertaking Progress Report (1989).

[4] van der Beken, H., IEEE Trans. NS-34 (1987) 742.

[5] Bombi F. et al. - The JET Project: Scientific and Technical Developments 1977-1978, EUR-6831 EN/EUR-JET-R1O (European Atomic Energy Community) 1980.

[6] Steed C.A. et al., Proc. 12th Symp. on Fusion Engineering, Vol. 1 (1987) p. 673: IEEE Cat. No. $87 \mathrm{CH} 2507-2$.

[7] van der Beken H. et al., IEEE Trans. NS-36 (1989) 1639

[8] Cohen D., Computer (Oct. 1981).

[9] GNU Bull. (The Free Software Foundation, Inc., Cambridge, USA).

\title{
Accelerator Control Systems
}

\section{P.N. Clout}

\author{
Vista Control Systems, Inc., Los Alamos, USA
}

Fig. 1 - Control rooms of CERN's accelerators. (a - left) For the PS machine in the mid-1960's where operators were obliged to move between racks of equipment in order to operate and adjust the machine.

(b - right) For the SPS machine in the late 1970's where the controls of the computer-based control system are grouped within reach of one operator around each of the several operator stations with their video screens, alphanumeric displays, oscilloscopes and devices for selecting and adjusting components of the accelerator.
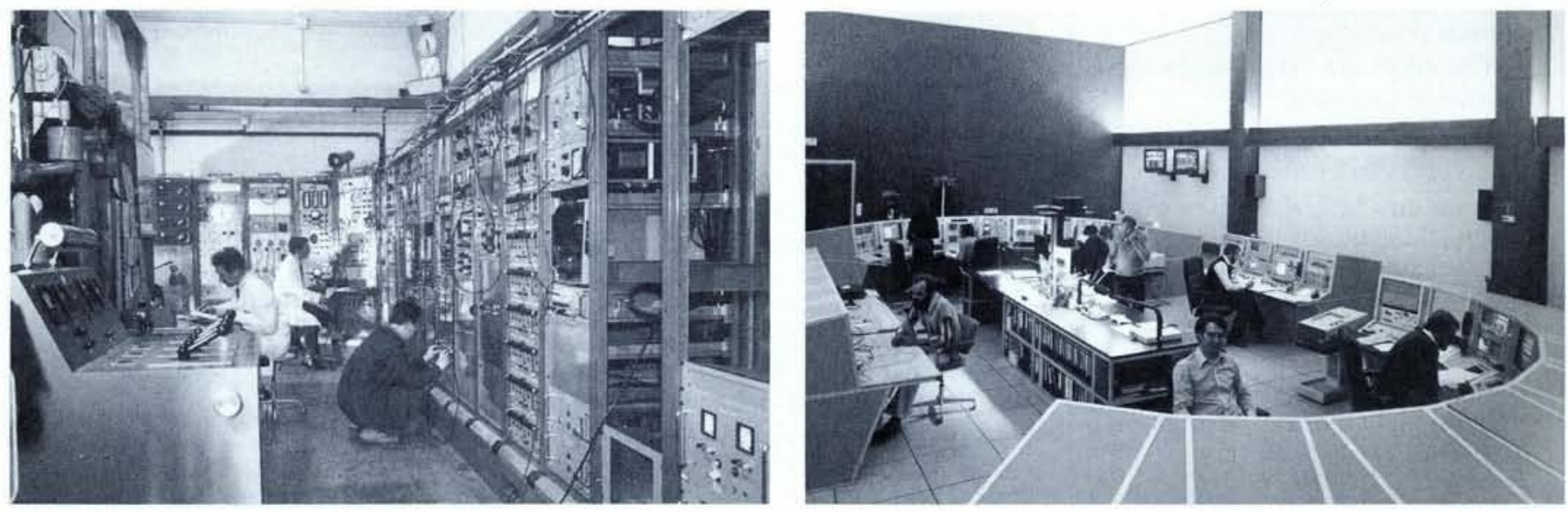

Charged particle accelerators were first connected to a computer in the early 1960's. The integration of computers with these research machines has expanded rapidly ever since, enabling considerable increases in size and performance.

Interest developed from what was probably the first reported use of a computer for data acquisition in nuclear physics by Edwin Norbeck in 1962 [1]. The report, and the rapid reduction in price of small computers, caught the attention of accelerator builders.

On entering an accelerator control room in the mid-1960's (Fig. 1) one would have 
seen racks of instrumentation directly connected to the devices either in equipment areas (power supplies, RF systems, etc.) or on the beam line (diagnostics, vacuum instrumentation, etc.). An example is the control room shown in Fig. 1a where the operator had to move among racks to run the accelerator.

A need for space in the control room for individual control panels arose as accelerators became larger, making operations and machine physics experiments difficult. Computers were first used for individual instruments and a number of approaches were adopted. The Argonne National Laboratory pioneered the field by adding a computer-based monitoring system to the ZGS accelerator in 1964: the Los Alamos National Laboratory followed with a computer control system which was essential for operating the LAMPF accelerator with its 10000 channels [2]. The LAMPF system also emphasized having all information and controls within the reach of one operator by multiplexing signals to general purpose devices at consoles - a concept that was also developed for CERN's very

Peter Clout received his Doctorate in physics from the University of York, UK in 1969. In 1989, he founded and became President of Vista Control Systems, Inc., 127 Eastgate Drive, Los Alamos, NM 87544, USA after working for nine years at the Los Alamos $\mathrm{Na}$ tional Laboratory where he was charged with developing and applying control systems. much larger SPS accelerator (Fig. 1b) and subsequently for many other facilities.

Other applications of computers developed in the USA and in Europe, encouraged by the small but growing use of computers for industrial process control, so by 1970 a proposal for a new accelerator invariably included a computer-based control system.

\section{Special Requirements}

If control systems for large charged particle accelerators are not fundamentally different to those for industrial processes, then conservative, well tested, commercial products can be used in place of risky, generally hand-crafted, and relatively expensive systems. The primary feature that distinguishes the two is that accelerators are effectively research projects in their own right since each experiment aims to develop specific beam properties. The requirements placed on the control system over the life of an accelerator are therefore unpredictable; considerable flexibility is sought. Timing features and diagnostic requirements also separate accelerators from many industrial processes.

\section{Timing features}

Characteristic time intervals in an accelerator range, at the low end, from some tens of picoseconds given by the length of a bunch of particles formed by accelerating, or bunching, RF frequencies of $\approx$ $1 \mathrm{GHz}$. At the high end, times of minutes and hours arise for the filling of a storage ring and the circulation of the stored beam around the ring. Two separate timing control systems are usually used to ensure synchronous operation. The RF reference system keeping the different RF generators in phase has the highest performance (to within a few degrees of phase of the RF frequency, although more recent systems work to a higher accuracy); it also often provides RF reference signals to the beam instrumentation. The other timing system coordinates the overall operation of the accelerator and is required to produce timing and gating pulses fully adjustable by the operator. Its accuracy is related to the circulation time of the beam and is usually about a microsecond, with timing being regulated up to minutes.

Another common requirement is the ability to operate an accelerator in a different way for each pulse: a full cycle is made up by selecting various sets of these settings (see page 31). A machine like LAMPF that operated at $120 \mathrm{~Hz}$ could be shared between experiments that "saw" different beams with different repetition rates. LAMPF's timing system with its 120 sets of timing patterns was further developed for CERN's PS machine where each cycle of a "supercycle" can have characteristic analog settings for magnets, RF cavities, etc., as well as different timing patterns. This was more practical at the PS as a cycle lasts a few seconds or so rather than for LAMPF's 8.3 milliseconds.

\section{VSYSTEM...}

\section{Seeing The Future In Data Acquisition And Control}

Vsystem is the total solution for data acquisition and control. Vsystem gives you network-oriented, flexible control system software with an advanced graphical interface.

\section{Networked, Real-time Database}

The Vsystem database is a memory-resident global section providing excellent performance. You can define the Vsystem database from an ASCII file generated directly with a text editor or as a report from a simple database management system.

\section{Reduced Development Time}

A custom system for a medium to large scale data acquisition/control system can require many manyears of effort to design, write and test before any application programming can be performed. With the aid of Vsystem you will dramatically decrease the time needed to develop your data acquisition and control system.

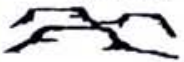

For Brochures and Information Contact:

\section{- Dynamic Graphics}

Vsystem, running on an Xwindows graphics device, provides easy prototyping of advanced graphical interfaces. You can also create mimic diagrams and graphical symbols.

Once you have created your display with the sophisticated drawing toolbox, you can activate the display and animate interfaces in real-time. Vsystem even provides the flexibility of being able to dynamically modify a control screen.

\section{- Flexible Input/Output Systems}

- Full Data-logging Capabilities

- Interactive Alarm Module

- Integrated Sequence Language

\section{- Currently Runs Under VAX/VMS and VAX/ELN}

Vista Control Systems, Inc., 127 Eastgate Drive, \#30800, Los Alamos, NM 87544, USA, (505) 662-2484, FAX (505) 662-3956 European Distributor: Hytec Electronics Ltd., Ladbroke Close, Woodley, Reading, Berks RG5 4DX, England, +44 (734) 6979 73, FAX ++44 (734) 441068

Agent: L-Data, Ch. de Somais 9, 1009 Pully, Switzerland, ++41 (21) 2981 43, FAX ++41 (21) 283668 


\section{Diagnostics}

Operators and machine physicists must know basic properties of the beam at many points. It is often impossible to measure anything but gross features, and then only at relatively few points owing to cost and space limitations, and because any measurement often necessarily uses an invasive technique (wire scanner, phosphor screen, video camera) which perturbs the beam. Each diagnostic device is a small physics experiment, usually with complex electronic processing of analogue signals before digitization for reading by the control system's computer. These diagnostics can take several years to develop and are generally tailored to a machine.

\section{The 1970's}

\section{Highways and databases}

The 1970's saw a number of developments that are represented by several control systems. On the hardware side, acceptance of the CAMAC standard and the availability of the CAMAC serial highway and basic software support greatly simplified the development of control system hardware while reducing costs and risk. The Hahn Meitner Institute (HMI) in Berlin was an early user of the serial highway and its system based on a single computer (a PDP-11), being ideal for smallto medium-sized implementations, was copied by a number of other institutes. It used a database for configuring control parameters instead of having the CAMAC addresses and other parameter-related information scattered all over the various programs, a concept that has been steadily developed elsewhere (see page 35 ).

\section{Networks}

A weakness of the single computer approach showed up when the requirements expanded beyond the computer's capabilities. Several computers linked together were needed, but it was necessary to develop one's own computer network as generalized computer networks were not commercially available at the time. Several laboratories implemented systems using a hierarchy of computers, but relying on a single computer as the focus for the controls.

The network hardware always comprised serial links between two computers. These links ranged from terminal lines to specially designed systems. CERN's SPS system used a single computer as the message router for the whole system so any computer could access any other an important advantage that was offset by the need for reliability provided by a back-up to the message routing computer. This system was therefore a functionally equivalent predecessor to today's bus and ring networks where messages are passed between control computers around rings or communications buses (see e.g. page 23, Fig. 2).

\section{Computer languages}

The SPS control system also introduced another element into the accelerator control field, namely interpreters for the application programs - a development that reflected the need for an evolving control system as the demands changed and understanding of the accelerator improved. In simple terms, an interpreter is a program that is able to error check, compile (convert to code) and execute operator instructions formulated using straightforward, structured commands (the alternative is to compile, link and execute in separate steps). The NODAL interpreter was developed specifically for a multicomputer environment. Based on FOCAL (FOrmula CALulator), it was extended to include input/output and the ability to send program code to another computer to be interpreted there and to communicate data in return. The SPS controls group was then free to concentrate on the basic control system and the application programming environment, leaving the actual application programming to engineers, physicists and operators.

In spite of problems with interpreters, NODAL was widely copied. Some laboratories adopted conventional languages, many of which have since fallen into disuse.

\section{Operator interface}

Developments of the operator interface in the 1970's included selection devices, graphics and programmable knobs for analog input. Among the selection devices experimented with to replace keyboard commands, the light pen, in contrast to track balls and touch screens, is now seldom seen. Touch screens to simplify the procedure the operator had to follow to indicate his next choice to the control system were developed by the Stanford Linear Accelerator Center (SLAC) and CERN for dedicated selection. The choices (CERN's SPS had 16 per level and SLAC's accelerator 64) were arranged as a selection hierarchy which the computer would draw on a video monitor: the next choices were presented whenever the operator indicated a choice until the selection was complete. The SPS system was copied by many laboratories and became commercially available. LAMPF employed a system of buttons where the legend card that was inserted to label the buttons was also machine-readable so that the operator made most of the selection by choosing the appropriate card.

The development of graphics for the presentation of data was started at this time, but with dedicated graphic screens. Most data were shown on alphanumeric video monitors and graphics displays were only successful for schematic diagrams where the limitation of one foreground and one background colour per character cell is unimportant.
The programmable knob simulating the control knob of a dedicated device by using a computer as an intermediary was developed to give a natural feel, and to allow the operator to observe several monitoring data while adjusting the knob. The concept was extended by including algorithms to adjust machine parameters that were not directly related to a single analogue output from the control system.

Thus, on entering an accelerator control room in the late 1970's (Fig. 1b) one would see several operator stations which were simplified considerably through the full exploition of computers. Each possessed a programme or parameter selection device (a video screen with a touch panel), a character-based computer monitor with alphanumeric data displays and schematics and possibly a track ball, and at least one computer-based control device. There would also have been at least one oscilloscope and some dedicated controls and indicators. However, this impressive overall result needed much work by many experts in control systems.

\section{The 1980's}

The developments of the 1970's were expanded in the early 1980's: computer networks and inexpensive computer workstations with excellent graphics support became readily available by 1985 ; the broad acceptance of computing standards began to allow the greater sharing of developments; the introduction of the Xerox Parc style of computing (a form of windowing software) started to influence accelerator control systems. These trends greatly simplified the implementation and further development of a control system.

Historically, the cost of a control system's software had been many times greater than the cost of the computers and the other hardware. For many accelerator projects, the availability of computer programmers was the limiting factor. The 1980's thus saw the rationalization of the design of the software structure, notably in the PS controls upgrade at CERN [3], the PSR project [4] and the NPB Telescope project [5] at Los Alamos. The inclusion of modern computer networks and workstations in a control system is epitomized by CERN's LEP machine, commissioned in 1989, that is noted for its large size.

However, for device interfacing the accelerator controls field diversified and became confused as groups chose different bus systems and inter-crate communications systems from among the many new standards that were promoted.

On a more positive note, control software for research projects was launched commercially in 1989. It draws upon the latest developments by operating under a specific type of computer display format called $X$ Window that facilitates the 
Fig. 2-An example of a computer display produced by one of the latest types of easy-to-use software that generates graphical control displays and data viewing windows in a multi-computer, networked control system for medium-tolarge physics experiments. It shows some of the objects that are employed to build up control windows graphically.

The window at the bottom left is the tool-box window of the graphical editor. This is used to

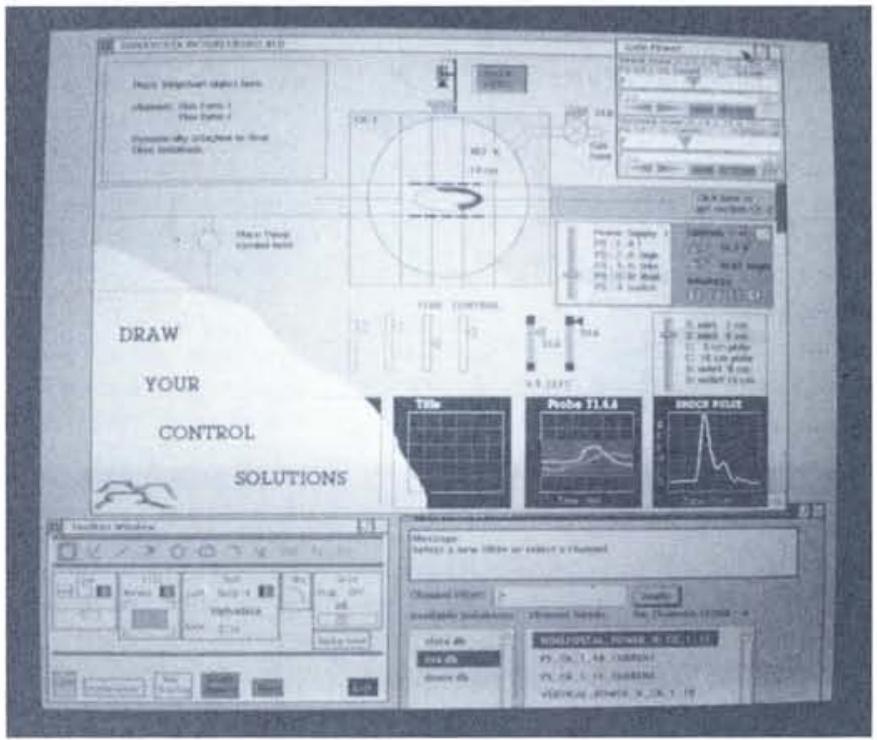

create the background drawing and to define and place the objects in the window that will be animated by connection to the real-time database. Other objects and active areas can be defined to control the windows and the overall operation of the control system.

At the upper right is a small slider bank which allows for the display and control of analogue values. Selection switches, bars and graphs can also be seen. Many other tools are available for use from the tool-box. In the bottom right is a separate window for viewing and changing the real-time databases, which include e.g. the channel descriptions. creation of graphical control displays and data viewing windows in a multi-computer, networked system (Fig. 2).

\section{Conclusions}

Computing is developing to provide the user with a more homogeneous working environment. This is also true for accelerator control systems, which are destined in the 1990's to become more integrated with physics design and simulation. This will allow greater understanding and automation of accelerators, resulting in advances in accelerator science and technology with reduced cost and risk.

\section{REFERENCES}

[1] Norbeck E., Proc. Conf. on the Utilization of Multiparameter Analyzers in Nuclear Physics, Grosinger, NY, 1962, p. 56.

[2] Puttnam T.M., Jameson R.A. and Schultheis T.M., IEEE Trans. NS-12 (1965) 21.

[3] Daneels A. and Skarek P., Nucl. Inst. Methods A247 (1986) 141.

[4] Clout P. N. et al., Nucl. Inst. Methods A247 (1986) 116.

[5] Clout P.N., Nucl. Inst. and Methods A293 (1990) 456 .

\section{Pulse-To-Pulse Modulation}

\section{R. Steiner \\ GSI, Darmstadt, Germany}

Associate Member of EPS

Accelerator performance for bunched particle beams has been enhanced significantly by modifying the beam properties on a pulse-to-pulse basis.

For continuous operation, a pulse is defined as the complete acceleration cycle in which the the properties of a bunch of charged particles of the same kind are changed. The pulse-to-pulse modulation (PPM) technique allows the uninterrupted production of beams of different particles with different characteristics in a single machine. It was, to the best of my knowledge, applied [1] for the first time in CERN's Proton Synchrotron complex (see overleaf).

Pulse-to-pulse-modulation is a powerful means to optimize the efficiency of pulsed accelerators. Hence:

- PPM provides beams to more than one user at virtually the same time.

- Many accelerators serve as injectors for another machine which is, as a rule, both larger and slower than the injector by about one order of magnitude. An injector,

Rudolf Steiner has a Diploma in physics from the University of Mainz, Germany. $\mathrm{He}$ is the Project Leader of the SIS/ESR Control Systems Group at GSI, Darmstadt where he was formerly the head of the electronics laboratory. for instance, is typically occupied with injection into the next machine for some $10 \%$ of the time: PPM releases the remaining $90 \%$ of the injector's beam time for other experiments with beams that may even be different to the injection beam. - Accelerators used in medicine exploit PPM for optimal performance by ensuring equal dose deposition in tissue.

Ideal PPM operation means that an arbitrary number of different acceleration cycles may be executed in an arbitrary sequence. For practical reasons, the number of different cycles is limited to a reasonable number, say 8 or 16 . The same is true for the "arbitrary" sequence: a limited number of cycles, say 256, forms a "supercycle" which is executed periodically. In spite of these restrictions, PPM has a considerable impact on the design of the control and timing systems of an accelerator facility.

\section{Impact on Equipment Control}

For PPM operation, the equipment control level (the lowest level in the control system) must be intelligent to provide flexibility, interrupt driven to provide speed and a precise response time, and equipped with enough memory to store the complete datasets of operating conditions (magnet ramps, etc.) of different cycles.
Security mechanisms must make sure that datasets are complete and consistent after operator interaction and before execution. Equipment controllers must be prepared to execute any legal function of a supercycle at any legal time. They must respond to coded interrupts, called events, as the number of legal functions will exceed the number of interrupt levels of the processors. An event may be a code (e.g. a 16-bit number) together with an interrupt request signal: it must contain information about the action to be performed (e.g. "start cycle"), the type of cycle, and eventually the supercycle.

Providing the complex, time dependent variations in the operating state needed for PPM on the equipment control level in pure hardware would be troublesome so intelligence must inevitably be placed at the local level.

\section{Impact on the Timing System}

A timing system is needed to generate events and to distribute them to equipment controllers. A bus system (see page 35), instead of the provision of individual signals for hundreds of separate pieces of equipment, is the most convenient approach for distributing events as the latter is inflexible and requires too much hardware. The events must occur in an orderly sequence: the timing system must only start actions that can be executed without conflicts. Once again, this can hardly be achieved without local intelligence.

\section{Conflicts}

However, the more flexible a timing system, the more sensitive it is to conflict 